\title{
Perfil lipídico de quatro espécies de peixes da região pantaneira de Mato Grosso do Sul
}

\author{
Lipid profile of four species of fish from the pantanal region of Mato Grosso do Sul
}

\author{
Manoel Mendes RAMOS FILHO ${ }^{1 *}$, Maria Isabel Lima RAMOS ${ }^{1}$, \\ Priscila Aiko HIANE ${ }^{1}$, Elizabeth Maria Talá de SOUZA²
}

\section{Resumo}

Os rios brasileiros possuem grande diversidade de peixes, no entanto, pouco se conhece sobre o potencial nutricional da maioria das espécies. O presente estudo avalia a composição centesimal e o perfil de ácidos graxos do tecido muscular dos peixes: pintado (Pseudoplatystoma coruscans), cachara (Pseudoplatystoma fasciatum), pacu (Piaractus mesopotamicus) e dourado (Salminus maxillosus), nativos do Rio Miranda na região do Pantanal Sul-Mato-Grossense. O peixe dourado mostrou o maior teor de proteína $(21,12 \%)$, enquanto o pintado o menor teor (17,90\%), não havendo diferença significativa entre teores de proteínas dos peixes cachara e pacu (cerca de $18 \%)$. O filé de pacu revelou ser o mais calórico, mesmo quando considerada a amostra seca. Nas quatro espécies de peixes, o ácido oléico foi predominante (20,25 a 37,25\%), seguido do ácido palmítico (19,96 a 21,37\%) e esteárico (7,39 a 9,82\%). O somatório dos teores do diferentes ácidos graxos poliinsaturados variou de 5,24 no pacu a 17,33\% no pintado, e dos ácidos graxos saturados de 32,91 a 38,89\%. As espécies cachara, pintado e dourado mostraram igual proporção de ácidos $\omega$-3 (média 7,80\%) e de ácidos $\omega$-6 (média 8,40\%), enquanto o pacu mostrou os menores teores (1,13 e $4,11 \%)$, respectivamente. Todas as amostras estudadas mostraram os índices $\omega-6 / \omega-3$ e hipocolesterolêmicos/hipercolesterolêmicos (HH) favoráveis quanto à qualidade nutricional. Apenas o pacu apresentou níveis não recomendados em relação aos índices de Trombogenicidade (IT) e poliinsaturados/saturados (P/S).

Palavras-chave: peixes; composição centesimal; ácidos graxos; ômega-3; lipídios.

\begin{abstract}
Brazilian rivers have a great diversity of fish, however, little is known about the nutritional potential of most native fish. The present study evaluates the centesimal composition and the fatty acid profile of the muscle tissue of four species of fish: pintado (Pseudoplatystoma coruscans), cachara (Pseudoplatystoma fasciatum), pacu (Piaractus mesopotamicus) and dourado (Salminus maxillosus), from the Miranda River in the pantanal region of Mato Grosso do Sul. Dourado had the highest protein content (21.12\%), while pintado had the lowest value (17.90\%). There was no difference between the protein levels of cachara and pacu (approximately 18\%). Pacu fillets were revealed to be the most caloric and this value did not change when the dry sample was considered. The four fish species presented oleic acid (20.25 to $37.25 \%)$ as the predominant fatty acid, followed by palmitic acid (19.96 to $21.37 \%$ ) and stearic acid (7.39 to $9.82 \%$ ). Total polyunsaturated fatty acid contents varied from 5.24 in pacu to $17.33 \%$ in pintado; and total saturated fatty acids from 32.91 to $38.89 \%$. Cachara, pintado and dourado species presented equal proportions of polyunsaturated fatty acids of the $\omega-3$ family (average of $7.80 \%$ ) and of the $\omega-6$ family (average of $8.40 \%$ ), while pacu presented the lowest contents of these fatty acids ( 1.13 and $4.11 \%$, respectively). All the samples studied presented $\omega 6 / \omega 3$ and hypocholesterolemic/hypercholesterolemic $(\mathrm{HH})$ indexes that indicate good nutritional quality. Only pacu presented non-recommended levels in relation to trombogenicity (TI) and polyunsaturated/saturated (P/S) indexes.

Keywords: fishes; proximate composition; fatty acids; omega-3; lipids.
\end{abstract}

\section{Introdução}

O pescado é um alimento importante na dieta de inúmeros grupos populacionais, não apenas como fonte de proteínas de alta qualidade nutricional, mas ainda como reserva significativa de ácidos graxos poliinsaturados da série ômega $3(\omega-3)$, aos quais são atribuídos numerosos benefícios à saúde humana (BADOLATO et al., 1994; ÇELIK; DILER; KÜÇÜKGÜLMEZ, 2005; VILA NOVA; GODOY; ALDRIGUE, 2005). Os ácidos $\omega$-3 e $\omega$-6 são precursores dos ácidos eicosanóides (prostaglandinas, tromboxanas e leucotrienos) e são essencialmente fornecidos pela dieta, uma vez que não são sintetizados pelo organismo humano. O ácido linoléico $(\omega-6)$ origina o ácido araquidônico, que é o precursor de eicosanóides tromboxanos e a prostaciclina $I_{2}$, respectivamente promotor e inibidor da agregação plaquetária. O ácido graxo alfa-linolênico ( $\omega$-3), precursor dos ácidos eicosapentaenóico (EPA) e docosahexaenóico (DHA), os quais além da função no desenvolvimento e funcionamento do sistema nervoso, fotorecepção e sistema reprodutivo (ÇELIK; DILER; KÜÇÜKGÜLMEZ, 2005; SIDHU, 2003; TAPIERO et al., 2002) são apontados como redutores de risco de doenças coronarianas, hipertensão moderada, incidência de diabetes e prevenção de certas arritmias cardíacas e morte súbita.

Recebido para publicação em 19/1/2007

Aceito para publicação em 30/7/2007 (002218)

${ }^{1}$ Departamento de Tecnologia de Alimentos e Saúde Pública, Universidade Federal de Mato Grosso do Sul - UFMS, Cidade Universitária, s/n, CP 549, CEP 79070-900,

Campo Grande - MS, Brasil, E-mail: mmramosf@nin.ufms.br

2 Departamento de Biologia Celular, Universidade de Brasília - UnB, Brasília - DF, Brasil

${ }^{*}$ A quem a correspondência deve ser enviada 
A constatação epidemiológica de que o consumo de peixes é capaz de reduzir riscos de doenças coronarianas situa a demanda de pescado nos países em desenvolvimento, não apenas como alternativa alimentar de alto valor nutritivo, mas ainda de consumo de um alimento funcional abundante. Em 2002, a produção brasileira de pescado atingiu 1.006.869 toneladas, com a participação da pesca extrativa continental de aproximadamente $24 \%$. A região Centro-Oeste produziu 11.946 toneladas e o Mato Grosso do Sul 4.744 toneladas de pescado oriundo do extrativismo, das quais $70,2 \%$ foram de peixes cachara, dourado, pacu e pintado (BRASIL, 2004). O presente estudo determinou a composição centesimal (umidade, proteínas, lipídios totais e cinzas) e o perfil de ácidos graxos da fração lipídica do tecido muscular (filé) de quatro espécies de peixes do Rio Miranda, na região do Pantanal Sul-Mato-Grossense, pintado (Pseudoplatystoma coruscans), cachara (Pseudoplatystoma fasciatum), pacu (Piaractus mesopotamicus) e dourado (Salminus maxillosus).

\section{Material e métodos}

\subsection{Amostras de peixes}

Os espécimes de peixe pintado Pseudoplatystoma coruscans $(\mathrm{n}=3)$, cachara Pseudoplatystoma fasciatum $(\mathrm{n}=3)$, pacu Piaractus mesopotamicus $(\mathrm{n}=3$ ) e dourado Salminus maxillosus $(\mathrm{n}=3)$ foram capturados no Rio Miranda, nas proximidades da Base de Estudos do Pantanal da Universidade Federal de Mato Grosso do Sul - UFMS, durante o período permitido para pesca, e acondicionados em caixas isotérmicas com gelo para o transporte. Cada amostra foi individualmente pesada e eviscerada, sendo a pele, as escamas e os espinhos separados do tecido muscular. Este último foi triturado e homogeneizado por triturador-homogeneizador elétrico.

\subsection{Análises de composição centesimal}

O tecido muscular homogeneizado foi imediatamente analisado em triplicata quanto ao teor de umidade $\left(105^{\circ} \mathrm{C}\right)$, resíduo mineral fixo (cinzas) por incineração em mufla a $550^{\circ} \mathrm{C}$ e teor de proteína total por método de Kjeldahl (AOAC, 1992). A extração de lipídio total foi realizada por extração a frio de acordo com Bligh e Dyer (1959), empregando-se as proporções recomendadas entre os solventes metanol e clorofórmio. A quantificação de lipídio total foi realizada gravimetricamente por evaporação a vácuo e a fração lipídica remanescente acondicionada a $-20^{\circ} \mathrm{C}$, em frasco âmbar sob atmosfera de nitrogênio para posterior análise.

\subsection{Composição dos ácidos graxos por cromatografia}

A fração lipídica total foi submetida à saponificação com $\mathrm{KOH}$ metanólico, seguida de esterificação com mistura de $\mathrm{H}_{2} \mathrm{SO}_{4}$ e $\mathrm{NH}_{4} \mathrm{Cl}$ em metanol e transferida para hexano, segundo descrito por Maia e Rodriguez-Amaya (1993). A análise dos ésteres metílicos dos ácidos graxos foi realizada em cromatógrafo gasoso (Varian, modelo Star 3400), equipado com detector de ionização de chama, injetor "Split/splitless", coluna capilar de sílica fundida contendo polietilenoglicol como fase estacionária (DB-Wax, $30 \mathrm{~m} \times 0,25 \mathrm{~mm}$, J\&W Scientific), nas seguintes condições cromatográficas: temperatura do injetor $250{ }^{\circ} \mathrm{C}$; temperatura da coluna $180{ }^{\circ} \mathrm{C}$ durante 20 minutos, programada a $2{ }^{\circ} \mathrm{C}$ por minuto até $220{ }^{\circ} \mathrm{C}$; temperatura do detector $260{ }^{\circ} \mathrm{C}$, gás de arraste hidrogênio com fluxo de $1,1 \mathrm{~mL} / \mathrm{min}$., gás "make-up" nitrogênio a $22 \mathrm{~mL} / \mathrm{min}$. e volume de injeção de $0,5 \mu \mathrm{L}$. Para a identificação dos ácidos graxos compararam-se os tempos de retenção com os dos padrões ésteres metílicos (Sigma-Aldrich), enquanto a quantificação foi realizada pela normalização de área expressando-se o resultado em percentual de área de cada ácido sobre a área total de ácidos graxos (\%). A transformação da porcentagem de área para g. $100 \mathrm{~g}^{-1}$ de tecido muscular foi feita multiplicando a área correspondente a cada ácido (\%) pelo teor da fração lipídica total e fatores de conversão para peixes magros e gordos, dividindo este resultado por 100, segundo Holland et al. (1994).

\section{4 Índices da qualidade nutricional (IQN) dos lipídios}

A qualidade nutricional da fração lipídica foi avaliada por três índices a partir dos dados de composição em ácidos graxos, através dos seguintes cálculos: Índice de Aterogenicidade $(\mathrm{IA})=[(\mathrm{C} 12: 0+(4 \times \mathrm{C} 14: 0)+\mathrm{C} 16: 0)] /(\Sigma \mathrm{AGMI}+\Sigma \omega 6+\Sigma \omega 3) ;$ Índice de Trombogenicidade $(\mathrm{IT})=(\mathrm{C} 14: 0+\mathrm{C} 16: 0+\mathrm{C} 18: 0) /$ $[(0,5 \times \Sigma$ AGMI $)+(0,5 \times \Sigma \omega 6+(3 \times \Sigma \omega 3)+(\Sigma \omega 3 / \Sigma \omega 6)]$, segundo Ulbricth e Southgate (1991); e razão entre ácidos graxos hipocolesterolêmicos e hipercolesterolêmicos $(\mathrm{HH})=$

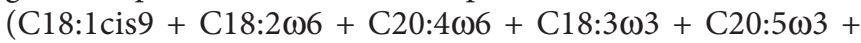

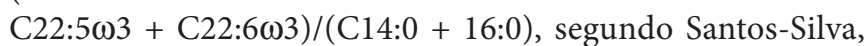
Bessa, Santos-Silva, (2002). Em que: AGMI = todos os ácidos monoinsaturados.

\subsection{Análise estatística}

Os valores da composição centesimal e da composição de ácidos graxos foram submetidos à análise de variância (ANOVA). O teste de Tukey foi aplicado para as variâncias desiguais entre os valores médios das amostras. Utilizou-se software Bioestat, versão 4.0, mantendo-se o nível de significância de 5\% em todas as análises.

\section{Resultados e discussão}

Os filés dos peixes analisados mostraram valores diferenciados em sua composição química (Tabela 1). O teor de umidade variou de $59,85 \%$ no pacu a $77,26 \%$ no pintado, enquanto que o menor conteúdo de cinzas foi observado no cachara $(0,76 \%)$ e o maior no dourado (1,18\%). Embora o conteúdo médio de proteína das quatro espécies estudadas tenha ficado dentro de uma faixa relativamente estreita, entre 17,90 e $21,12 \%$, a comparação indicou diferenças significativas, exceto entre o pacu e o cachara. Teores de umidade, mineral e proteínas na mesma faixa observada no presente estudo foram encontrados no músculo de espécies de água doce como o curimbatá (Prochilodus scrofa), tilápia (Oreochromis niloticus) e mapará (Hypophthalmus edentatus) (MAIA; RODRIGUEZ-AMAYA; FRANCO, 1994; OLIVEIRA; AGOSTINHO; MATSUSHITA, 2003; VILA NOVA; GODOY; ALDRIGUE, 2005).

O tecido muscular do pacu mostrou a maior concentração de lipídios em relação à composição total $(19,83 \pm 0,12 \%)$, 
seguido do cachara $(10,03 \pm 0,63 \%)$, pintado $(3,30 \pm 0,24 \%)$ e dourado (2,64 $\pm 0,11 \%)$. Resultados que, segundo Ackman (1989), classificam as duas primeiras espécies de peixes como alimento de alto teor de gordura ( $>8 \%)$, enquanto o pintado e o dourado ficam na categoria de baixo teor de gordura (entre 2 a 4\%). O pintado e o dourado pela classificação de Stansby e Olcott (1967) podem ser considerados na categoria A $(<5 \%$ de lipídios e proteína entre 15 e 20\%). Andrade et al. (1995) também relataram em peixes de água doce no sul do país (Maringá - PR, Brasil), um conteúdo baixo de lipídios no filé de dourado e pintado, e alto no pacu (Colossoma mitrei), valores de 0,88 , 1,27 e 18,31\%, respectivamente. Gutierrez e Silva (1993) comparando o conteúdo lipídico de sete espécies de peixes de água doce oriundos de rios brasileiros encontraram um menor teor de lipídios $(0,41 \%)$ no filé de pintado.

Tabela 1. Composição centesimal do tecido muscular do filé de cachara, pintado, pacu e dourado expressa em g. $100 \mathrm{~g}^{-1}$ de músculo.

\begin{tabular}{lcccr}
\hline \multicolumn{1}{c}{ Peixes } & $\begin{array}{c}\text { Cachara } \\
\text { média }^{*} \pm d p\end{array}$ & $\begin{array}{c}\text { Pintado } \\
\text { média }^{*} \mathrm{dp}\end{array}$ & $\begin{array}{c}\text { Pacu } \\
\text { média }^{*} \mathrm{dp}\end{array}$ & $\begin{array}{c}\text { Dourado } \\
\text { média* }^{*} \mathrm{dp}\end{array}$ \\
\hline Umidade & $70,58 \pm 0,67^{\mathrm{a}}$ & $77,26 \pm 1,16^{\mathrm{b}}$ & $59,85 \pm 0,35^{\mathrm{c}}$ & $75,01 \pm 0,34^{\mathrm{d}}$ \\
Proteínas & $18,50 \pm 0,63^{\mathrm{a}}$ & $17,90 \pm 0,35^{\mathrm{b}}$ & $18,89 \pm 0,24^{\mathrm{a}}$ & $21,12 \pm 0,22^{\mathrm{c}}$ \\
Lipídios totais & $10,03 \pm 0,63^{\mathrm{a}}$ & $3,30 \pm 0,24^{\mathrm{b}}$ & $19,83 \pm 0,12^{\mathrm{c}}$ & $2,64 \pm 0,11^{\mathrm{d}}$ \\
Mineral fixo & $0,76 \pm 0,05^{\mathrm{a}}$ & $1,01 \pm 0,03^{\mathrm{b}}$ & $0,92 \pm 0,01^{\mathrm{c}}$ & $1,18 \pm 0,01^{\mathrm{d}}$ \\
\hline${ }^{*}$ Média de triplicata \pm desvio padrão $(\mathrm{dp}) ;$ e valores na mesma linha seguidos de letras \\
iguais não diferem entre si $(\mathrm{p}<0,05)$.
\end{tabular}

Nos quatro tipos de peixes analisados houve predominância de ácidos graxos insaturados em relação ao total de lipídios (50,22 a 53,43\%), com maior concentração de monoinsaturados variando entre 33,81 a 47,53\% (Tabela 2). Embora o teor de ácidos monoinsaturados no pacu $(47,53 \%)$ tenha sido significativamente superior aos demais peixes analisados, o conteúdo médio de poliinsaturados de $5,24 \%$ nesta espécie foi o menor $(\mathrm{p}=0,0006)$.

A análise da composição de ácidos graxos (Tabela 2) evidenciou que o ácido oléico (C18:1 $\omega$-9) seguido do palmítico (C16:0) e em menor proporção do esteárico (C18:0) foram os mais abundantes nos quatro diferentes peixes, com teores variando respectivamente de 20,25 a 37,25, 19,96 a 21,27 e 7,39 a $9,82 \%$ do total de ácidos graxos. Dentre as espécies analisadas, o pacu revelou o maior conteúdo de ácido oléico (37,25\%), e no cachara o maior conteúdo foi o de ácido palmítico (21,37\%). O predomínio desses ácidos parece ser característico de peixes de água doce; de fato Andrade et al. (1995) encontraram em filés de dourado (Salminus maxillosus) os valores de 9,76, 39,44 e 9,25\% de ácido oléico, palmítico e esteárico, respectivamente. Recentemente, Luzia et al. (2003) mostraram predominância do ácido palmítico em tilápia (Oreochromis spp.) 35,9\% e curimbatá (Prochilodus spp.) 28,9\%.

O pintado evidenciou alto conteúdo de ácidos graxos poliinsaturados (17,33\% do total de ácidos graxos), todavia

Tabela 2. Composição de ácidos graxos do tecido muscular do filé de cachara, pintado, pacu e dourado, expressos em \% de área relativa ao total de ácidos graxos e em g de ácido graxo. $100 \mathrm{~g}^{-1}$ de tecido muscular.

\begin{tabular}{|c|c|c|c|c|c|c|c|c|}
\hline \multirow[t]{2}{*}{ Ácidos graxos } & \multicolumn{2}{|c|}{ Cachara } & \multicolumn{2}{|c|}{ Pintado } & \multicolumn{2}{|c|}{ Pacu } & \multicolumn{2}{|c|}{ Dourado } \\
\hline & $\%$ & g. $100 \mathrm{~g}^{-1}$ & $\%$ & g. $100 \mathrm{~g}^{-1}$ & $\%$ & g. $100 \mathrm{~g}^{-1}$ & $\%$ & g. $100 \mathrm{~g}^{-1}$ \\
\hline C12:0 & $0,00 \pm 0,00$ & $0,00 \pm 0,00$ & $0,00 \pm 0,00$ & $0,00 \pm 0,00$ & $2,62 \pm 0,46^{\mathrm{a}}$ & $0,46 \pm 0,08^{\alpha}$ & $0,63 \pm 0,06^{\mathrm{b}}$ & $0,01 \pm 0,00^{\beta}$ \\
\hline C14:0 & $1,79 \pm 0,21^{\mathrm{ac}}$ & $0,16 \pm 0,02^{\alpha}$ & $1,34 \pm 0,03^{\mathrm{a}}$ & $0,03 \pm 0,00^{\alpha}$ & $5,73 \pm 0,78^{b}$ & $1,02 \pm 0,13^{\beta}$ & $3,08 \pm 0,03^{c}$ & $0,06 \pm 0,00^{\alpha}$ \\
\hline C14: $1 \omega-5$ & $0,90 \pm 0,15^{\mathrm{a}}$ & $0,08 \pm 0,01^{\alpha}$ & $0,68 \pm 0,13^{\mathrm{ac}}$ & $0,02 \pm 0,00^{\beta}$ & $0,06 \pm 0,01^{\mathrm{b}}$ & $0,01 \pm 0,00^{\beta}$ & $0,41 \pm 0,00^{c}$ & $0,01 \pm 0,00^{\beta}$ \\
\hline C16:0 & $21,37 \pm 0,86^{\mathrm{a}}$ & $1,93 \pm 0,07^{\alpha}$ & $20,82 \pm 0,83^{\mathrm{a}}$ & $0,48 \pm 0,02^{\beta}$ & $19,96 \pm 0,30^{\mathrm{a}}$ & $3,56 \pm 0,05^{\gamma}$ & $20,94 \pm 0,53^{a}$ & $0,39 \pm 0,01^{\beta}$ \\
\hline $\mathrm{C} 16: 1 \omega-7$ & $5,57 \pm 0,31^{\mathrm{a}}$ & $0,50 \pm 0,02^{\alpha}$ & $5,74 \pm 0,33^{\mathrm{a}}$ & $0,13 \pm 0,01^{\beta}$ & $5,25 \pm 0,38^{a}$ & $0,94 \pm 0,06^{\gamma}$ & $7,70 \pm 0,01^{\mathrm{b}}$ & $0,14 \pm 0,00^{\beta}$ \\
\hline $\mathrm{C} 18: 1 \omega-9$ & $25,48 \pm 1,19^{a}$ & $2,30 \pm 0,11^{\alpha}$ & $23,30 \pm 1,68^{\mathrm{ac}}$ & $0,54 \pm 0,04^{\beta}$ & $37,25 \pm 1,25^{b}$ & $6,64 \pm 0,22^{\gamma}$ & $20,25 \pm 0,85^{\mathrm{c}}$ & $0,37 \pm 0,01^{\beta}$ \\
\hline C18:1 $\omega-7$ & $4,25 \pm 0,04^{\mathrm{a}}$ & $0,38 \pm 0,00^{\alpha}$ & $4,26 \pm 0,73^{\mathrm{a}}$ & $0,10 \pm 0,02^{\beta}$ & $3,62 \pm 0,18^{a}$ & $0,64 \pm 0,03^{\gamma}$ & $3,81 \pm 0,15^{\mathrm{a}}$ & $0,07 \pm 0,00^{\beta}$ \\
\hline $\mathrm{C} 18: 2$ cis $\omega-6$ & $5,92 \pm 0,80^{\mathrm{a}}$ & $0,53 \pm 0,07^{\alpha}$ & $4,77 \pm 1,16^{\mathrm{abc}}$ & $0,11 \pm 0,02^{\beta}$ & $2,64 \pm 0,43^{b}$ & $0,47 \pm 0,08^{\alpha}$ & $5,98 \pm 0,04^{\mathrm{ac}}$ & $0,11 \pm 0,00^{\beta}$ \\
\hline $\mathrm{C} 18: 3 \omega-3$ & $4,48 \pm 1,07^{\mathrm{a}}$ & $0,41 \pm 0,10^{\alpha}$ & $1,98 \pm 0,18^{\mathrm{bc}}$ & $0,05 \pm 0,00^{\beta}$ & $0,72 \pm 0,15^{\mathrm{b}}$ & $0,13 \pm 0,03^{\beta}$ & $3,34 \pm 0,08^{\mathrm{ac}}$ & $0,06 \pm 0,00^{\beta}$ \\
\hline C20:0 & $0,47 \pm 0,05^{\mathrm{a}}$ & $0,04 \pm 0,00^{\alpha}$ & $0,50 \pm 0,05^{\mathrm{a}}$ & $0,01 \pm 0,00^{\beta}$ & $0,15 \pm 0,03^{b}$ & $0,02 \pm 0,00^{\gamma}$ & $0,25 \pm 0,04^{\mathrm{b}}$ & $0,01 \pm 0,00^{\beta}$ \\
\hline C22:0 & $0,39 \pm 0,04^{\mathrm{a}}$ & $0,03 \pm 0,00^{\alpha}$ & $0,35 \pm 0,01^{\mathrm{a}}$ & $0,01 \pm 0,00^{\beta}$ & $0,00 \pm 0,00$ & $0,00 \pm 0,00$ & $0,00 \pm 0,00$ & $0,00 \pm 0,00$ \\
\hline C22:1 $\omega-9$ & $0,20 \pm 0,00$ & $0,18 \pm 0,00$ & $0,00 \pm 0,00$ & $0,00 \pm 0,00$ & $0,00 \pm 0,00$ & $0,00 \pm 0,00$ & $0,00 \pm 0,00$ & $0,00 \pm 0,00$ \\
\hline $\mathrm{C} 22: 6 \omega-3$ & $1,78 \pm 0,17^{\mathrm{ac}}$ & $0,16 \pm 0,01^{\alpha}$ & $5,64 \pm 1,45^{\mathrm{b}}$ & $0,13 \pm 0,03^{\alpha \gamma}$ & $0,32 \pm 0,12^{\mathrm{a}}$ & $0,06 \pm 0,02^{\beta}$ & $3,43 \pm 0,64^{\mathrm{bc}}$ & $0,06 \pm 0,01^{\beta \gamma}$ \\
\hline$\sum$ saturados & $34,27 \pm 0,92^{\mathrm{a}}$ & $3,09 \pm 0,08^{\alpha}$ & $33,12 \pm 1,81^{\mathrm{a}}$ & $0,77 \pm 0,04^{\beta}$ & $38,89 \pm 1,27^{b}$ & $6,93 \pm 0,23^{\gamma}$ & $32,91 \pm 0,80^{\mathrm{a}}$ & $0,61 \pm 0,02^{\beta}$ \\
\hline $\begin{array}{l}\sum \text { monoinsaturados } \\
\text { (AGMI) }\end{array}$ & $38,06 \pm 1,14^{\mathrm{a}}$ & $3,44 \pm 0,10^{\alpha}$ & $36,10 \pm 1,92^{\mathrm{a}}$ & $0,83 \pm 0,05^{\beta}$ & $47,53 \pm 1,60^{b}$ & $8,47 \pm 0,29^{\gamma}$ & $33,81 \pm 1,14^{\mathrm{a}}$ & $0,62 \pm 0,02^{\beta}$ \\
\hline $\begin{array}{l}\sum \text { poliinsaturados } \\
\text { (AGPI) }\end{array}$ & $14,99 \pm 1,30^{\mathrm{a}}$ & $1,35 \pm 0,12^{\alpha}$ & $17,33 \pm 2,69^{a}$ & $0,40 \pm 0,06^{\beta}$ & $5,24 \pm 0,74^{b}$ & $0,93 \pm 0,13^{\gamma}$ & $16,41 \pm 1,26^{\mathrm{a}}$ & $0,30 \pm 0,02^{\beta}$ \\
\hline$\sum \omega-6$ & $8,12 \pm 0,85^{\mathrm{a}}$ & $0,73 \pm 0,07^{\alpha}$ & $8,43 \pm 1,24^{\mathrm{a}}$ & $0,19 \pm 0,03^{\beta}$ & $4,11 \pm 0,48^{\mathrm{b}}$ & $0,73 \pm 0,09^{\alpha}$ & $8,77 \pm 0,47^{\mathrm{a}}$ & $0,16 \pm 0,01^{\beta}$ \\
\hline$\sum \omega-3$ & $6,87 \pm 1,05^{\mathrm{a}}$ & $0,62 \pm 0,10^{\alpha}$ & $8,90 \pm 1,80^{\mathrm{a}}$ & $0,20 \pm 0,04^{\beta}$ & $1,13 \pm 0,28^{\mathrm{b}}$ & $0,20 \pm 0,05^{\beta}$ & $7,63 \pm 0,78^{a}$ & $0,14 \pm 0,02^{\beta}$ \\
\hline
\end{tabular}

Valores médios \pm desvio padrão (dp); valores expressos em \%, na mesma linha, seguidos de letras iguais, não diferem entre si ( $\mathrm{p}<0,05)$; valores expressos em g.100 $\mathrm{g}^{-1}$, na mesma linha, seguidos de letras gregas iguais não diferem entre si $(\mathrm{p}<0,05)$; AGMI = Ácidos Graxos Monoinsaturados; e AGPI = Ácidos Graxos Poliinsaturados. 
esse valor não mostrou diferença significativa $(p>0,05)$ ao teor observado no cachara e dourado. Percentuais mais altos, de 20,66 e $30,93 \%$ de ácidos poliinsaturados no pintado e dourado, respectivamente, foram encontrados por Andrade et al. (1995), no entanto, o teor de lipídio total quantificado por estes autores foi de apenas 1,27\% para o pintado e $0,88 \%$ para o dourado, inferiores, portanto, ao determinado no presente estudo. Variação na composição química de peixes pode ocorrer em função de vários fatores endógenos e exógenos, dentre eles: genética, tamanho, sexo, estágio reprodutivo, alimentação, fatores ambientais, temperatura e estação do ano (LUZIA et al., 2003; MOREIRA et al., 2001).

A análise específica do conteúdo de ácidos graxos essenciais (Tabela 2) mostra que os ácidos linoléico (C18:2 cis $\omega 6)$, linolênico (C18:3 $\omega 3)$, araquidônico (C20:4 $\omega 6)$, eicosapentaenóico (EPA, 20:5 $\omega 3$ ) e docosahexaenóico (DHA, C22:6 $\omega$ 3) representaram conteúdo inferior a $6 \%$ do total de ácidos graxos. O ácido linoléico foi o ácido essencial predominante nas espécies cachara, pacu e dourado enquanto o DHA predominou no pintado $(5,64 \%)$. A predominância do ácido linoléico também foi observada em diferentes espécies de água doce, atingindo um percentual máximo de 21,92\% (ANDRADE et al., 1995; MOREIRA et al., 2002). Em peixes de rios brasileiros valores mais altos foram encontrados por Andrade et al. (1995) na corvina (Plagioscion squamosissimus) e jurupoca (Hemisorubim plathyrhinchos), respectivamente para o EPA $(11,67 \%)$ e DHA (14,99\%). Em amostras de peixes Brycon, Moreira et al. (2001) observaram teor máximo de DHA $(1,60 \%)$ e semelhante ao valor do cachara (máximo de $1,78 \pm 0,17 \%$ ).

A qualidade nutricional do perfil lipídico avaliada por diferentes índices encontra-se descrita na Tabela 3. Alimentos que apresentam a razão ácidos graxos poliinsaturados e saturados (P/S) abaixo de 0,45 têm sido considerados como indesejáveis à dieta (DEPARTMENT OF HEALTH AND SOCIAL SECURITY, 1984) por sua potencialidade na indução do aumento de colesterol sanguíneo. Nos peixes estudados esta razão variou de 0,13 no pacu até 0,52 no pintado, no entanto, o índice $\mathrm{P} / \mathrm{S}$ avaliado isoladamente tem recebido restrições, uma vez que não considera os efeitos metabólicos dos ácidos graxos monoiinsaturados (WILLIAMS, 2000).

Considerando a razão $\omega 6 / \omega 3$, valores abaixo de 4,0 sugerem quantidades desejáveis à dieta para a prevenção de riscos cardiovasculares (DEPARTMENT OF HEALTH AND SOCIAL SECURITY, 1994). A relação $\omega 6 / \omega 3$ variou de 0,95 no filé do pintado a 3,65 no pacu, resultados que promovem todos os peixes estudados à categoria de potencialmente saudáveis. A

Tabela 3. Índices de qualidade nutricional da fração lipídica do filé de cachara, pintado, pacu e dourado.

\begin{tabular}{lccccc}
\hline & P/S & $\omega 6 / \omega 3$ & HH & IA & IT \\
\hline Cachara & 0,44 & 1,18 & 1,75 & 0,54 & 0,59 \\
Pintado & 0,52 & 0,95 & 1,84 & 0,49 & 0,33 \\
Pacu & 0,13 & 3,65 & 1,66 & 0,86 & 1,16 \\
Dourado & 0,49 & 1,14 & 1,49 & 0,70 & 0,35 \\
\hline
\end{tabular}

$\mathrm{P} / \mathrm{S}=$ Poliinsaturados/saturados; $\omega 6 / \omega 3=\Sigma$ da série Omega $6 / \Sigma$ da série Omega 3 ; $\mathrm{HH}=\Sigma$ hipocolesterolêmicos $/ \Sigma$ hipercolesterolêmicos; $\mathrm{IA}=$ índice de aterogenicidade; e IT = índice de trombogenicidade. (ULBRICHT; SOUTHGATE, 1991). razão $\omega 6 / \omega 3$ observada no presente estudo é inferior a de outras espécies de água doce relatadas por Moreira et al. (2001) (variação de 1,14 a 8,79).

O cálculo da razão $\Sigma$ ácidos graxos hipocolesterolêmicos/ $\Sigma$ ácidos graxos hipercolesterolêmicos, índice $(\mathrm{HH})$ relacionado mais especificamente com o metabolismo do colesterol, resultou em valores na faixa de 1,49 a 1,84 , inferiores ao intervalo de 2,03 a 2,46 encontrado para outros peixes de água doce ou salgada (TESTI et al., 2006). Valores altos para essa relação são desejáveis sob o ponto de vista nutricional.

O índice de aterogenicidade (IA), que relaciona os ácidos pró e antiaterogênicos no cachara, pintado, pacu e dourado foram respectivamente 0,$54 ; 0,49 ; 0,86$ e 0,70 . Valores com a mesma extensão foram encontrados por Senso et al. (2007) no peixe marinho Sparus aurata cultivado e estocado sob congelamento (faixa de 0,21 a 0,29 ). Em contraste à relação $\mathrm{HH}$, valores mais baixos para IA são desejáveis.

Dentre as considerações para avaliação da qualidade dietética de alimentos é incluída a razão $\omega 6 / \omega 3$ menor que 10 , ingestão de 0,3 a $0,5 \mathrm{~g} / \mathrm{dia}(2.000 \mathrm{kcal})$ para EPA + DHA e 0,8 a 1,0 g/dia para o ácido $\alpha$-linolênico (KRAUSS et al., 2000; KRIS-ETHERTON; HARRIS; APPEL, 2003). Neste contexto a ingestão de $100 \mathrm{~g}$ de filé de cachara representa $73 \%$ da recomendação mínima diária de EPA + DHA e $51 \%$ de $\alpha$-linolênico recomendado; as quatro espécies possuem conteúdo recomendado para a razão $\omega 6 / \omega 3 \mathrm{em} 100 \mathrm{~g}$ de tecido muscular. O conteúdo proposto como mínimo para o ácido $\alpha$-linolênico é alcançado por cerca de $195 \mathrm{~g}$ de filé de cachara.

\section{Conclusões}

- As espécies de peixes cachara, pacu, dourado e pintado mostraram ser boas fontes de proteínas, sendo que os dois primeiros também são ricos em lipídios;

- No total de ácidos graxos da fração lipídica da porção comestível dos peixes analisados foi encontrada maior porcentagem de ácidos insaturados, com predominância de monoinsaturados; e

- Na avaliação da qualidade nutricional dos lipídios, todas as amostras estudadas mostraram os índices $\omega 6 / \omega 3$ e HH favoráveis quanto ao consumo alimentar; no entanto em relação aos índices IT e P/S, o pacu apresentou níveis não recomendados.

\section{Referências bibliográficas}

ACKMAN, R. G. Nutritional composition of fats in seafoods. Progress in Food and Nutrition Science, Oxford, v. 13, n. 1 p. 161-241, 1989.

ANDRADE, A. D. et al. w3 fatty acids in freshwater fish from south Brazil. Journal of the American Oil Chemists' Society, Champaign, v. 72, n. 10, 1, p. 1207-1210, 1995.

AOAC - Association of Official Analytical Chemists. Official methods of analysis. $12^{\text {th }} \mathrm{ed}$. Washington, 1992. $1115 \mathrm{p}$.

BADOLATO, E. S. G. et al. Composição centesimal de ácidos graxos e valor calórico de cinco espécies de peixes marinhos nas diferentes 
estações do ano. Revista do Instituto Adolfo Lutz, São Paulo, v. 54, n. 1, p. 27-35, 1994.

BLIGH, E. G.; DYER, W. J. A rapid method of total lipid extraction and purification. Canadian Journal of Biochemistry and Physiology, Ottawa, v. 37, n. 8, p. 911-917, 1959.

BRASIL. Ministério do Meio Ambiente. Instituto Brasileiro do Meio Ambiente e dos Recursos Naturais Renováveis - IBAMA. Estatística da pesca 2002, Brasil, Grandes Regiões e Unidades da Federação. Tamandaré-PE, 2004. Disponível em: <http://www.ibama.gov.br/ rec_pesqueiros/download.php?id_download=40>. Acesso em: 09 nov. 2004.

ÇELIK, M.; DILER, A.; KÜÇÜKGÜLMEZ, A. A comparison of the proximate compositions and fatty acid profiles of zander (Sander lucioperca) from two different regions and climatic conditions. Food Chemistry, Champaign, v. 92, n. 4, p. 637-641, 2005.

DEPARTMENT OF HEALTH AND SOCIAL SECURITY. Diet and cardiovascular disease. Report on Health and Social Subjects, n. 28. London: HMSO, 1984.

Nutritional aspects of cardiovascular disease. Report on Health and Social Subjects, n.46. London: HMSO, 1994. 178 p.

GUTIERREZ, L. E.; SILVA, R. C. M. Fatty composition of commercially important fish from Brazil. Scientia Agricola, Piracicaba, v. 50, n. 3, p. 478-483, 1993.

HOLLAND. B. et al. The composition of foods. Cambridge, UK: Mc Cance and Widdowson's, 1994. p. 8-9.

KRAUSS, R. M. et al. American Heart Association Dietary Guidelines Revision 2000: A Statement for Healthcare Professionals From the Nutrition Committee of the American Heart Association. Circulation, Dallas, v. 102, n. 18, p. 2284-2299, 2000.

KRIS-ETHERTON, P. M.; HARRIS, W. S.; APPEL, L. J. Omega-3 fatty acids and cardiovascular disease: New recommendations from the American Heart Association. Arteriosclerosis Thrombosis and Vascular Biology, Dallas, v. 3, n. 2, p.151-152, 2003.

LUZIA, L. A. et al. The influence of season on the lipid profiles of five commercially important species of Brazilian fish. Food Chemistry, Champaign, v. 83, n. 1, p. 93-97, 2003.

MAIA, E. L.; RODRIGUEZ-AMAYA, D. B.; FRANCO, M. R. B. Fatty acids of the total, neutral, and phospholipids of the brazilian freshwater fish Prochilodus scrofa. Journal of Food Composition and Analysis, London, v. 7, n. 4, p. 240-251, 1994.

MAIA, E. L.; RODRIGUEZ-AMAYA, D. B. Avaliação de um método simples e econômico para a metilação de ácidos graxos com lipídios de diversas espécies de peixes. Revista do Instituto Adolfo Lutz, São Paulo, v. 53, n. 1/2, p. 27-35, 1993.

MOREIRA, A. B. et al. Fatty acids profile and cholesterol contents of three brazilian Brycon freshwater fishes. Journal of Food Composition and Analysis, London, v. 14, n. 6, p. 565-574, 2001.

Oliveira, E. R. N.; AGOstinho, A. A.; MATsushitA, M. Effect of biological variables and capture period on the proximate composition and fatty acid composition of the dorsal muscle tissue of Hypophtalmus edentatus (Spix, 1829). Brazilian Archives of Biology and Technology, Curitiba, v. 46, n. 1, p. 105-114, 2003.

SANTOS-SILVA, J.; BESSA, R. J. B.; SANTOS-SILVA, F. Effect of genotype, feeding system and slaughter weigt on the quality of light lambs. II. Fatty acid composition of meat. Livestock Production Science, Roma, v. 77, n. 2/3, p. 187-194, 2002.

SENSO, I. et al. On the possible effects of harvesting season and chilled storage on the fatty acid profile of the fillet of farmed gilthead sea bream (Sparus aurata). Food Chemistry, Champaign, v. 101, n. 1, p.298-307, 2007. Disponível em: <http://www.sciencedirect.com>. Acesso em: 08 ago. 2006.

SIDHU, K. S. Health benefits and potential risks related to consumption of fish or fish oil. Regulatory Toxicology and Pharmacology, New York, v. 38, n. 3, p. 336-344, 2003.

STANSBY, M. E.; OLCOTT, H. S. Composición del pescado. In: STANSBY, M. E.; DASSOW, J. A. Tecnología de la Industria Pesquera. Zaragoza: Acribia, 1967. p. 391-402.

TAPIERO, H. et al. Polynsaturated fatty acids (PUFA) and eicosanoids in human health and pathologies. Biomedicine and Pharmacotherapy, Paris, v. 56, n. 5, p. 215-222, 2002.

TESTI, S. et al. Nutritional traits of dorsal and ventral fillets from three farmed fish species. Food Chemistry, Champaign, v. 98, n. 1, p. 104-111, 2006.

ULBRICHT, T. L. V.; SOUTHGATE, D. A. T. Coronary heart disease: seven dietary factors. Lancet, London, v. 338, n. 8773, p. 985-992, 1991.

VILA NOVA, C. M. V. M.; GODOY, H. T.; ALDRIGUE, M. L. Composição química, teor de colesterol e caracterização dos lipídios totais de tilápia (Oreochromis niloticus) e pargo (Lutjanus purpureus). Ciência e Tecnologia de Alimentos, Campinas, v. 25, n. 3, p. 430-436, 2005.

WILLIAMS, C. M. Dietary fatty acids and human health. Annales de Zootechnie, Paris, v. 49, n. 3, p. 165-180, 2000. 